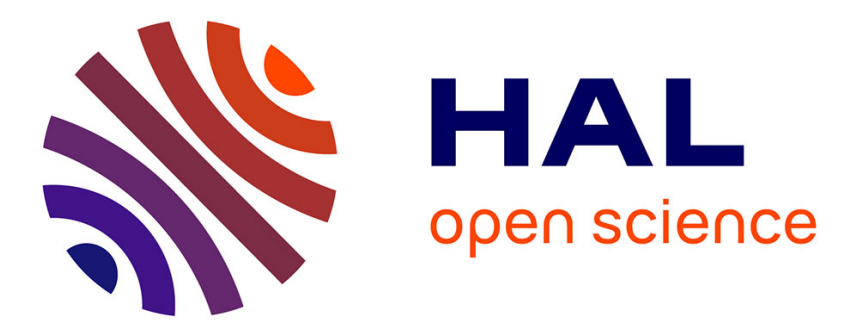

\title{
Acceleration of land surface model development over a decade of glass
}

B. van den Hurk, M. Best, P. Dirmeyer, A. Pitman, Jan Polcher, J. Santanello

\section{To cite this version:}

B. van den Hurk, M. Best, P. Dirmeyer, A. Pitman, Jan Polcher, et al.. Acceleration of land surface model development over a decade of glass. Bulletin of the American Meteorological Society, 2011, 92 (12), pp.1593-1600. 10.1175/BAMS-D-11-00007.1 . hal-01134121

\section{HAL Id: hal-01134121 \\ https://hal.science/hal-01134121}

Submitted on 23 Mar 2015

HAL is a multi-disciplinary open access archive for the deposit and dissemination of scientific research documents, whether they are published or not. The documents may come from teaching and research institutions in France or abroad, or from public or private research centers.
L'archive ouverte pluridisciplinaire HAL, est destinée au dépôt et à la diffusion de documents scientifiques de niveau recherche, publiés ou non, émanant des établissements d'enseignement et de recherche français ou étrangers, des laboratoires publics ou privés. 


\section{ACCELERATION OF LAND SURFACE MODEL DEVELOPMENT OVER A DECADE OF GLASS}

by Bart van den Hurk, Martin Best, Paul Dirmeyer, Andy Pitman, Jan Polcher, and Joe Santanello

The Global Land Atmosphere System Study has ushered in an era in which LSMs for numerical weather and climate prediction now incorporate complex vegetation responses, detailed hydrology, dynamic snowpack evolution, urban processes, and more.

$\mathrm{L}$ and surface models (LSMs) used in numerical weather prediction and climate projections have seen considerable development since the early simple "bucket scheme" of Manabe (1969). From the pioneering work by Deardorff (1978), the development of globally applicable LSMs by Dickinson et al. (1986) and Sellers et al. (1986) and the building of the first models that represent vegetation dynamics (e.g., Foley et al. 1996), LSMs now represent heterogeneity, complex vegetation responses to environmental

AFFILIATIONS: VAN DEN HURK-KNMI, De Bilt, Netherlands; Best-Met Office, Devon, United Kingdom; DIRMEYER-Center for Ocean-Land-Atmosphere Studies, Calverton, Maryland; PitMan-Climate Change Research Centre, University of New South Wales, Sydney, New South Wales, Australia; POLCHERLaboratoire de Météorologie Dynamique du CNRS, Paris, France; SANTANELLO-NASA Hydrospheric and Biospheric Sciences Laboratory, Greenbelt, Maryland CORRESPONDING AUTHOR: Bart van den Hurk, P.O. Box 20I, KNMI De Bilt 3730AE, Netherlands

E-mail: hurkvd@knmi.nl

The abstract for this article can be found in this issue, following the table of contents.

DOI:10.1175/BAMS-D-II-00007.I

In final form 28 April 2011

(201I American Meteorological Society conditions, detailed surface and subsurface hydrology, dynamic evolution of snowpacks, and even representations of urban, lake, and biogeochemical processes. A thorough review of the present state of the art in land surface modeling would probably require tens if not hundreds of pages to address all of the relevant developments [see Levis (2010) for a recent review]. Here we present an overview of initiatives that are a part of the Global Land Atmosphere System Study (GLASS; available online at www .gewex.org/glass), ${ }^{1}$ including the antecedent community modeling efforts that led up to the formation of GLASS. Reference will be made to a number of projects in which GLASS is involved. An overview of these can be found in Table 1 .

There has long been recognition of the need to confront LSMs with observational data. However, in the early 1990s, Henderson-Sellers et al. (1993) appreciated the need to evaluate and intercompare LSMs within a common framework. She launched the Project for the Intercomparison of Land-Surface Parameterization Schemes [PILPS; the first model

${ }^{1}$ GLASS is one of the scientific panels under the umbrella of the Global Water and Energy Cycle Experiment (GEWEX), a core project of the World Climate Research Programme (WCRP). 
intercomparison project (MIP)], with the aim of improving the understanding of current and future parameterization schemes used to represent regional to continental scales. PILPS was sponsored by the World Meteorological Organization's Working Group on Numerical Experimentation (WGNE) and the GEWEX science panels. The first meeting was held in June 1992 in Columbia, Maryland. PILPS was singularly successful. Through the 1990s-2000s it coordinated multiple offline (uncoupled from atmospheric models) experiments, first with synthetic atmospheric forcing (Pitman et al. 1993) and later with observed forcing. The first of these (Chen et al. 1997; see Fig. 1) used data from the atmospheric boundary layer research station at Cabauw in the Netherlands to produce one of the most highly cited papers in land surface modeling and to establish the weaknesses inherent in the Manabe (1969) scheme, as well as the failure of many LSMs at that time to conserve energy and water. Increasingly well-constrained experiments followed, focused mainly on mid- and high-latitude regions. For example, Wood et al. (1998) and Liang et al. (1998) focused on the Red-Arkansas River basin in the central United States; Schlosser et al. (2000) and Slater et al. (2001) concentrated on the boreal grasslands in Valdai, Russia; and Nijssen et al. (2003) and Bowling et al. (2003) examined the Torne-Kalix basin in Sweden.

PILPS's significant and ongoing contribution has been to facilitate the testing and intercomparison of LSMs against point-based observational data. Many of the technical challenges that PILPS helped resolve are now commonly implemented in LSMs-issues such as the need to run LSMs decoupled from the host atmospheric model, and the recognition of the need to formally conserve energy and water. PILPS was also originally conceived to compare LSMs in the coupled environment. While efforts to examine the coupled behavior of LSMs were explored and some critical facilitating technologies were introduced [e.g., a Network Common Data Form (NetCDF) protocol for defining output variables and metadata, Assistance for Land-Surface Modelling Activities (ALMA); see Table 1], along with a common land surface coupler (Polcher et al. 1998), PILPS could not resolve the full spectrum of land surface challenges alone.

Growing in part from the International Satellite Land-Surface Climatology Project (ISLSCP), an effort was launched to derive $2 \mathrm{yr}$ of near-surface atmospheric forcing globally over all land surfaces except Antarctica (Meeson et al. 1995). The data were produced at a $1^{\circ}$ spatial resolution and were combined with observational datasets and global analyses from a global weather model to resolve the diurnal cycle. The Global Soil Wetness Project Phase 1 (GSWP-1; Dirmeyer et al. 1999) used the ISLSCP global data to drive LSMs in a framework similar to how they are used in weather and climate models. GSWP-1 was, in one sense, a global implementation of the point-based PILPS evaluations. However, it also had the aim of generating specific products of value. The gridded global atmospheric forcing datasets were technically challenging to develop, and many individual modeling groups found handling the quantity of data and performing the global simulations demanding. However, GSWP-1 was revolutionary in allowing a truly global evaluation of LSMs, encompassing all climate zones and capturing some degree of interannual variability. Comparison of basin-averaged hydrology highlighted the importance of high-quality rainfall forcing in order to simulate correctly the net discharge of water from land to the oceans (Oki et al. 1999). Soil wetness datasets produced in GSWP-1 were used in retrospective forecasts of seasonal climate to show that interannual variations of the land surface state have a significant impact on climate prediction (e.g., Dirmeyer 2000; Douville 2002).

TABLE I. Overview of GLASS projects.

\begin{tabular}{|l|l|l|}
\hline Acronym & \multicolumn{1}{|c|}{ Expansion } & \multicolumn{1}{|c|}{ Reference(s) } \\
\hline PILPS & Project for Intercomparison of Land-Surface Schemes & Pitman et al. (1993) and Chen et al. (1997) \\
\hline GSWP & Global Soil Wetness Project & Dirmeyer et al. (1999, 2006) \\
\hline ALMA & Assistance for Land-Surface Modelling Activities & www.Imd.jussieu.fr/ polcher/ALMA \\
\hline GLACE & Global Land Atmosphere Coupling Experiment & Koster et al. (2004, 2009) \\
\hline LDAS & Land Data Assimilation System & Rodell et al. (2004) \\
\hline LUCID & Land-Use and Climate, Identification of Robust Impacts & Pitman et al. (2009) \\
\hline LoCo & Local coupling & Van den Hurk and Blyth (2008) \\
\hline PILDAS & Project for Intercomparison of Land Data Assimilation Systems & \\
\hline PALS & Protocol for the Analysis of Land Surface models & www.pals.unsw.edu.au \\
\hline
\end{tabular}


Both PILPS and GSWP-1 were critical in bringing the LSM community together, one primarily at the point or catchment scale, and the other at the global scale. However, in isolation neither had the capacity to put their respective contribution into the larger perspective or spectrum of terrestrial processes spanning the point scale with uncoupled simulations to the global fully coupled simulations.

To address this challenge of a more holistic program around land surface processes, GLASS was launched in 1999 and was led by a panel tasked to accelerate the progress made by PILPS and GSWP-1. This panel continued these projects. GSWP was led into a second phase (GSWP-2), a dramatically extended research program covering a $10-y r$ period (Dirmeyer et al. 2006). GSWP-2 used a range of LSMs, numerous gridded forcing datasets, and a set of evaluation criteria as part of the protocol.

The results of GSWP-2 revealed that the variability in estimated global and annual mean evaporation over land from the participating LSMs is still considerable, and is in fact not a great deal smaller than the range in estimates one can find in the literature back to the start of the twentieth century (Schlosser and Houser 2007). The spread between LSMs can be understood from the fact that over time a wide range of LSMs have been developed for different host models: integrating these models outside of their native environments reveals different sensitivities to the common forcings. However, the projects have provided improved estimates of the typical interannual variability in land surface states and fluxes, uncertainties in observational datasets and reanalyses, climate-dependent model sensitivities, and regional energy and water balances. GSWP-2 also generated a global archive of "realistic" land surface states and fluxes that are used to evaluate the contribution of land processes to atmospheric and hydrologic variability. A thorough review of GSWP is given by Dirmeyer (2011).

To date, this discussion has reflected on LSMs uncoupled from a host model. A critical goal of GLASS was to expand from the uncoupled (or "offline") point-based (PILPS) and globally based (GSWP) evaluations to include simulations that are fully coupled with the atmosphere. Coupled simulations can include LSMs that are either fully integrated into a weather forecast or climate model or coupled into a common mesoscale atmospheric model (Santanello et al. 2009).

The essential contribution to date from the global coupled analysis relates to the Global Land Atmosphere Coupling Experiment (GLACE), a program of research led jointly with the Climate Variability

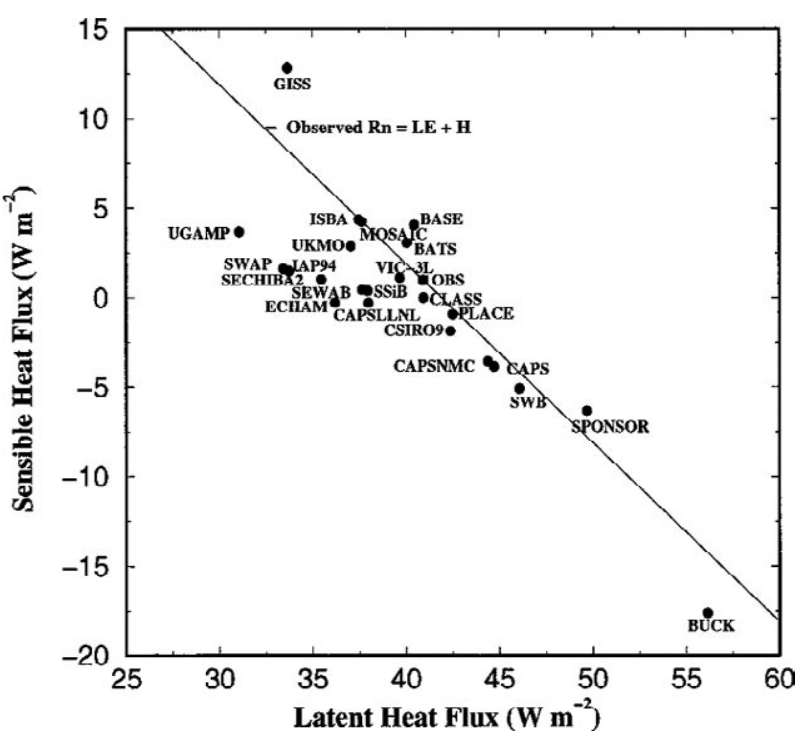

Fig. I. Example of PILPS scatterplot (from Chen et al. 1997).

(CLIVAR) panel of WCRP. GLASS helped formulate GLACE, which led to the fundamental discovery by Koster et al. (2004) that LSMs interact with their overlying atmospheric models with remarkably different coupling strengths. In some areas and under some conditions, the state of the land surface systematically affects the atmospheric variability, particularly temperature and rainfall. In the first GLACE experiment, highly controlled seasonal simulations with a dozen different weather and climate forecast models were conducted. The experiment was designed to isolate and quantify the impact of the land surface state, namely, soil wetness, on boreal summer climate variability. For the first time a multimodel map was produced showing areas where land-atmosphere interaction has the strongest effect on precipitation variability (Fig. 2). The considerable spread in this "coupling strength" between the models is often used to illustrate the lack of understanding of this complex coupling process. However, the overall picture that strong sensitivities appear in transitional climate regimes (between arid and humid regions) can be understood from basic physical arguments: near strong gradients of surface evaporation and precipitation, changing the link between soil moisture, evaporation, and precipitation is likely to change the precipitation variability. In the recent follow-up experiment GLACE-2 (Koster et al. 2010), the practical implication of this finding was examined by assessing the contribution of realistic land initial conditions to the prediction of precipitation and temperature. This new multimodel experiment demonstrated that increased skill can be expected on time scales beyond deterministic atmospheric 
Land-atmosphere coupling strength (JJA), averaged across AGCMs

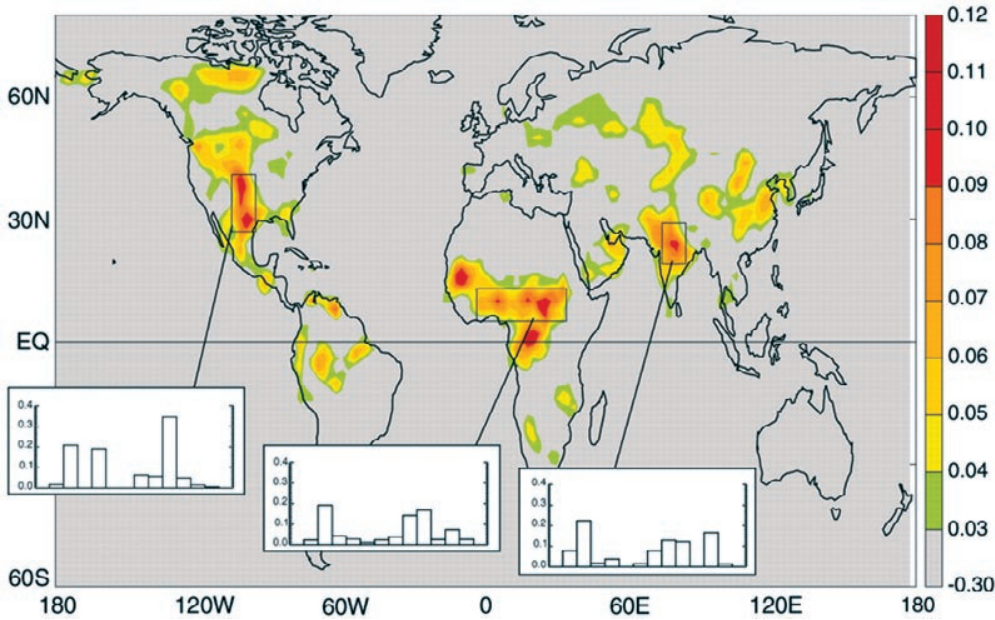

FIG. 2. Land-atmosphere coupling strength diagnostic (dimensionless) between modeled soil moisture and precipitation, determined in the GLACE experiment (Koster et al. 2004). [Reprinted with permission from AAAS.]

predictability (about 2 weeks) out to time scales where ocean-atmosphere interactions become the dominant forcing of climate variations (about 2 months). Forecast skill increases particularly in areas where the precipitation observations used to generate the initial soil moisture states (obtained from GSWP-2) are of high quality and gauge density. Also, it was found that stronger initial soil moisture anomalies lead to larger skill improvements.

To complement the global and seasonal climate focus of GLACE, the issue of land-atmosphere coupling at the process level (i.e., from local to regional) is systematically being addressed in the local coupling ("LoCo") theme (Van den Hurk and Blyth 2008). Land-atmosphere interactions are present at all scales. For instance, the atmospheric properties within a plant canopy directly change in response to fluxes to and from individual leaves. The atmospheric boundary layer feedback reduces evaporation from the surface after being moistened by earlier evaporation. Convection can be triggered by soil moisture anomalies, thereby reinforcing or reducing these very same anomalies (see Seneviratne et al. 2010). Because of this complex hierarchy of processes, and the strong interaction with ambient atmospheric conditions, a straightforward experimental design to systematically evaluate the degree to which land surface processes affect the atmosphere locally is not easily realized. However, a continuous scientific discussion engaged by a series of GLASS workshops led to an experimental protocol using a numerical land-atmosphere model "laboratory" where a wide range of land, boundary layer, and cloud models can be interchanged and subjected to meaningful diagnostics under controlled conditions [Land Information System-Weather Research and Forecasting (LIS-WRF)]. Newly developed diagnostics address the scale dependence and various natures of land-atmosphere feedback, and they include a combination of land and atmospheric variables (Santanello et al. 2009). LoCo is an example of a GLASS project where a fairly long incubation time was needed before a practical experimental design could be formulated (the first LoCo workshop was held in 2003); however, it will transform the ability of LSMs to realistically represent not only the fluxes and states, but also the complex interactions and feedbacks with the atmosphere. Inputs to these workshops were provided by colleagues from the GEWEX panels on boundary layers [Global Atmospheric Boundary Layer Study (GABLS; online at www.gewex.org /gabls.htm)] and clouds [Global Cloud System Study (GCSS; online at www.gewex.org/gcss.html)].

An important aspect of land modeling is the specification of the land surface characteristics and their temporal and spatial variability. The importance of this implementation is convincingly demonstrated by a recent study addressing the impact of land use change-the Land-Use and Climate, Identification of Robust Impacts (LUCID; Pitman et al. 2009). LUCID was a GLASS-Integrated Land-EcosystemAtmosphere Process Study (iLEAPS; online at www .ileaps.org/) in which seven GCMs were given a similar land use change scenario. A large part of the variability of the regional climate impact of land use change could be attributed to different assumptions on the change of LSM parameters associated with the imposed land use change. A systematic protocol to objectively assess the sensitivity of surface fluxes to the specification of canopy conductance, leaf area index, surface roughness, and rooting depth is not easily defined, resulting from the fact that these quantities are strongly intertwined with the core LSM structure. However, the current development of GCMs into sophisticated Earth system models (incorporating the biogeochemical cycles associated with the biotic components of our climate system) warrants a careful analysis of the role of these land surface characteristics. 
Since the inception of GLASS, the scientific LSM arena has seen rapid evolution. PILPS-type experiments have become integrated into land surface model development and diagnostics, and are now commonly performed for an expanding number of climate regimes and land-related process areas. Model-based global estimates are now being considered as a valuable component of climatologies of the land surface states and fluxes, demonstrated by activities around the LandFlux (http://wgdma .giss.nasa.gov/landflux.html) initiative, coorganized by the GEWEX Hydroclimate Panel (GHP; online at www.gewex.org/projects-ghp.html). Land Data Assimilation Systems (LDASs; Rodell et al. 2004) have been modeled after the GSWP framework, and all of the operational LDASs as well as most land surface intercomparison projects use the ALMA protocols. GLACE-like procedures and metrics are adopted in quite a few studies addressing land-atmosphere interaction, including changes in the patterns under future climate conditions (Seneviratne et al. 2006). Recognizing the importance of uncertainties in prescribed model parameters for model results and data assimilation products, parameter estimation tools and associated forecast evaluation diagnostics have been implemented in Land Information System (LIS; available online at http://lis.gsfc.nasa.gov). However, the overarching questions-how good should our land models be?, how accurately can we estimate land variables on a global scale?, or how large is the inherent climate predictability related to land?-still require new scientific approaches.

In this changing landscape, GLASS has recently restructured its scientific agenda, and is currently in the process of launching new concepts and experimental designs aimed at progressing land surface science. The original structure of GLASS was a two-by-two matrix, where one axis represented spatial scale (point/plot/catchment versus continental/global) and the other differentiated between uncoupled and coupled modeling. In the new structure, three core activities have been defined: benchmarking, model data fusion, and coupling (Fig. 3).

Benchmarking of LSMs (and datasets) urgently needs attention in the wider scientific community. Do we actually know what we can expect from the quality of models and datasets? In an inspiring experiment, Abramowitz et al. (2008) evaluated the skill of an LSM driven by and evaluated with data from a number of flux network (FluxNet; available online at http://daac.ornl.gov/FLUXNET /fluxnet.shtml) sites. Apart from the land models, an unrelated statistical model was calibrated on a subset of the observed forcings, and evaluated with an independent subset. In many ways, this statistical model considerably outperformed the state-of-the-art LSM simulations. This result leads to the conclusion that the complex physical equations embedded in the LSMs did not utilize the information content inherent in the forcing data well. These equations typically have many parameters, few of which can be practically optimized for most locations. For an LSM to be useful for predictions, it must be demonstrated that the model physics actually adds information to the prediction system. Thus, in our model evaluation experiments we should reduce model errors to a minimum, but also specify what the minimum acceptable error actually is. Obviously this depends on the application of the model. For example, a flood forecasting center only using modeled runoff to predict the occurrence of floods in a river basin has a different definition of the minimum acceptable error than scientists trying to attribute trends in evaporation to soil moisture processes (Jung et al. 2010). A general benchmark for models could be that they are able to capture a useful mode of variability (e.g., interannual variability, or match the error level of the validation observations), but more specific benchmarks need to be developed. GLASS seeks ways to engage and formalize this process. A good showcase for this is the proposed third phase of GSWP (online at http://hydro.iis.u-tokyo.ac.jp /HESSS2/), in which the earlier GSWP-2 datasets will be extended forward to the present, enabling scientific progress toward attribution of recent changes to various components of the climate system, including

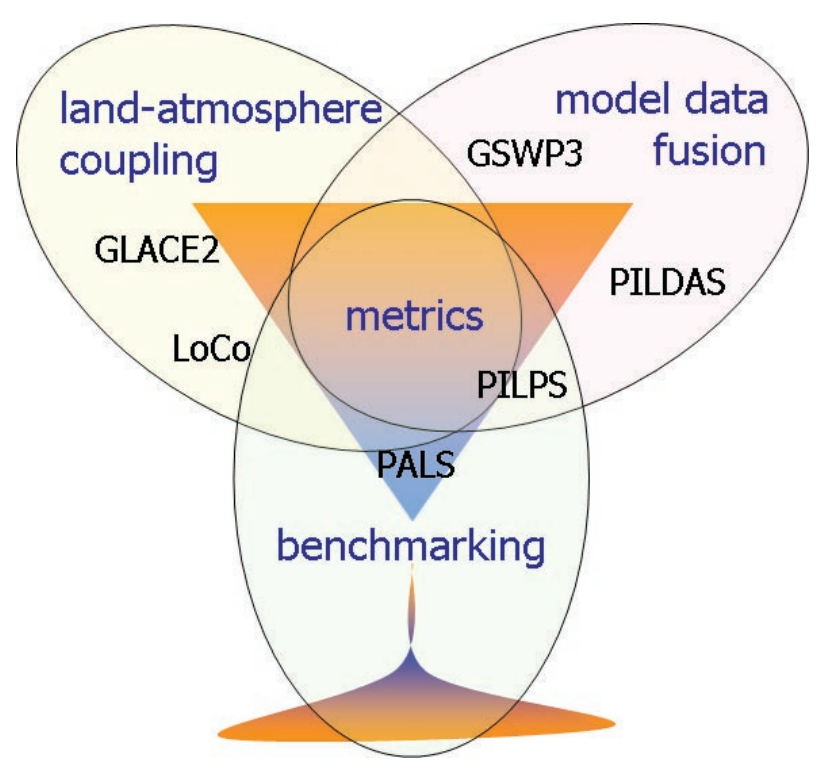

Fig. 3. Layout of new GLASS structure. 
the terrestrial component. The development of the web-based Protocol for Analysis of Land Surface Schemes (PALS; online at www.pals.unsw.edu.au/) will help for an objective definition of useful benchmarking standards.

The activities clustered around model data fusion address the need to gain experience in the areas of data assimilation and parameter estimation. In various scientific arenas surrounding the land modeling domain (numerical weather prediction, catchment hydrology, and ocean science), data assimilation is a common tool to estimate optimal states of the climate system by blending observations with models constrained by physical equations. Also, the notion that model parameters should show larger variability leads to a rethinking of the concept of fixed land models that are driven by fixed atmospheric forcings. However, data assimilation techniques are conceptually simple but mathematically quite complex, and small changes in the underlying error assumptions can lead to large differences in the results. A newly formulated Project for Intercomparison of Land Data Assimilation Systems (PILDAS) is a first attempt to learn how configuration differences among a number of current operational land data assimilation systems affect the resulting estimates. Like the early PILPS projects, PILDAS contains a hierarchy of levels with subsequently increasing numbers of degrees of freedom. In the first pilot phase, a synthetic (model produced) dataset will be assimilated in a range of configurations. Ultimately PILDAS will address consequences of choices of data types, ways of preprocessing data, and technical settings, such as length of assimilation windows, spatial correlations, and error structure. Results from the first PILDAS phase will appear in 2012.

The coupling theme will continue the earlier work related to GLACE and LoCo, concentrating on the development of adequate diagnostics for land-atmosphere coupling that can be verified with observations and the use of standard modeling software (LIS), where model settings can be easily controlled and evaluated. Pilot experiments are currently ongoing, and a call for participation from the broader community can be expected over the next few years. It should be noted that the GLASS themes are certainly not independent, and activities in benchmarking and model data fusion will need to be considered in both uncoupled and coupled frameworks.

During its existence, GLASS activities have strengthened and created many scientific networks, leading to scientific progress. Reflecting WCRP's emphasis to contribute to operational modeling centers, the National Centers for Environmental Prediction (NCEP), Japan Meteorological Agency (JMA), Met Office (UKMO), Météo-France, and European Centre for Medium-Range Weather Forecasts (ECMWF), among others, have used GLASS activities to improve their forecast models. For example, results from PILPS-2E by Van den Hurk and Viterbo (2003) have been formally included in the ECMWF model by Balsamo et al. (2009). De Rosnay et al. (2009) explore LSM-generated soil moisture fields in West Africa to prepare for routine assimilation of Soil Moisture Ocean Salinity mission (SMOS) data. Routine application of LDAS products is used in operational forecasts of NCEP and other centers worldwide. Building on earlier successes, GLASS will continue to support projects that extend the earlier frameworks, like GSWP-3 or ongoing or new PILPSlike experiments, and renew its focus on emerging topics like model data fusion and benchmarking. As before, GLASS will coordinate workshops, model studies, and analyses in order to strengthen or create the scientific networks that are needed to bring the representation of value-adding land surface modules in Earth System Models to a higher level.

\section{REFERENCES}

Abramowitz, G., R. Leuning, M. Clark, and A. J. Pitman, 2008: Evaluating the performance of land surface models. J. Climate, 21, 5468-5481.

Balsamo, G., P. Viterbo, A. Beljaars, B. J. J. M. van den Hurk, A. Betts, and K. Scipal, 2009: A revised hydrology for the ECMWF model: Verification from field site to terrestrial water storage and impact in the integrated forecast system. J. Hydrometeor., 10, 623-643.

Bowling, L. C., and Coauthors, 2003: Simulation of high latitude hydrological processes in the Torne-Kalix basin: PILPS phase 2(e) 1: Experiment description and summary intercomparisons. Global Planet. Change, 38, 1-30.

Chen, T. H., and Coauthors, 1997: Cabauw experimental results from the Project for Intercomparison of Land-Surface Parameterization Schemes. J. Climate, 10, 1194-1215.

Deardorff, J. W., 1978: Efficient prediction of ground surface temperature and moisture, with inclusion of a layer of vegetation. J. Geophys. Res., 83, 1889-1903.

De Rosnay, P., and Coauthors, 2009: AMMA Land Surface Model Intercomparison Experiment coupled to the Community Microwave Emission Model: ALMIP-MEM. J. Geophys. Res., 114, D05108, doi:10.1029/2008JD010724. 
Dickinson, R. E., A. Henderson-Sellers, P. J. Kennedy, and M. F. Wilson, 1986: Biosphere-Atmosphere Transfer Scheme (BATS) for the NCAR Community Climate Model. NCAR Tech. Note TN-275 + STR, $69 \mathrm{pp}$.

Dirmeyer, P. A., 2000: Using a global soil wetness dataset to improve seasonal climate simulation. J. Climate, 13, 2900-2922.

— , 2011: A history and review of the Global Soil Wetness Project (GSWP). J. Hydrometeor., 12, 729-749.

_- A. Dolman, and N. Sato, 1999: The pilot phase of the Global Soil Wetness Project. Bull. Amer. Meteor. Soc., 80, 851-878.

—, X. Gao, M. Zhao, Z. Guo, T. Oki, and N. Hanasaki, 2006: GSWP-2: Multimodel analysis and implications for our perception of the land surface. Bull. Amer. Meteor. Soc., 87, 1381-1397.

Douville, H., 2002: Influence of soil moisture on the Asian and African monsoons. Part II: Interannual variability. J. Climate, 15, 701-720.

Foley, J. A., I. C. Prentice, N. Ramankutty, S. Levis, D. Pollard, S. Sitch, and A. Haxeltine, 1996: An integrated biosphere model of land surface processes, terrestrial carbon balance, and vegetation dynamics. Global Biogeochem. Cycles, 10, 603-628.

Henderson-Sellers, A., Z. L. Yang, and R. E. Dickinson, 1993: The Project for Intercomparison of LandSurface Schemes (PILPS). Bull. Amer. Meteor. Soc., 74, 1335-1349.

—, A. J. Pitman, P. K. Love, P. Irannejad, and T. Chen, 1995: The Project for Intercomparison of Land Surface Parameterization Schemes (PILPS) phases 2 and 3. Bull. Amer. Meteor. Soc., 76, 489-503.

Jung, M., and Coauthors, 2010: Recent decline in the global land evapotranspiration trend due to limited moisture supply. Nature, 467, 951-954, doi:10.1038 /nature09396.

Koster, R. D., and Coauthors, 2004: Regions of strong coupling between soil moisture and precipitation. Science, 305, 1138-1140.

— - and Coauthors, 2010: Contribution of land surface initialization to subseasonal forecast skill: First results from a multi-model experiment. Geophys. Res. Lett., 37, L02402, doi:10.1029/2009GL041677.

Levis, S., 2010: Modeling vegetation and land use in models of the earth system. Wiley Interdiscip. Rev. Climate Change, 1, doi:10.1002/wcc.83.

Liang, X., and Coauthors, 1998: The Project for Intercomparison of Land-Surface Parameterization Schemes (PILPS) phase 2(c) Red-Arkansas River basin experiment: 2. Spatial and temporal analysis of energy fluxes. Global Planet. Change, 19, 137-159.
Manabe, S., 1969: The atmospheric circulation and hydrology of the earth's surface. Mon. Wea. Rev., 97, 739-774.

Meeson, B. W., F. E. Corprew, J. M. P. McManus, D. M. Myers, J. W. Closs, K. J. Sun, D. J. Sunday, and P. J. Sellers, 1995: ISLSCP Initiative I-Global Data Sets for Land-Atmosphere Models, 1987-1988. Vols. 1-5, NASA, CD-ROM.

Nijssen, B., and Coauthors, 2003: Simulation of high latitude hydrological processes in the Torne-Kalix basin: PILPS phase 2(e) 2: Comparison of model results with observations. Global Planet. Change, 38, 31-53.

Oki, T., T. Nishimura, and P. Dirmeyer, 1999: Assessment of annual runoff from land surface models using Total Runoff Integrating Pathways (TRIP). J Meteor. Soc. Japan, 77, 235-255.

Pitman, A. J., and Coauthors, 1999: Key results and implications from phase 1 (c) of the Project for the Intercomparison of Land-Surface Parameterization Schemes. Climate Dyn., 15, 673-684.

— , and Coauthors, 2009: Uncertainties in climate responses to past land cover change: First results from the LUCID intercomparison study. Geophys. Res. Lett., 36, L14814, doi:10.1029/2009GL039076.

Polcher, J., and Coauthors, 1998: A proposal for a general interface between land-surface schemes and general circulation models. Global Planet. Change, 19, 261-276.

Rodell, M., and Coauthors, 2004: The global land data assimilation system. Bull. Amer. Meteor. Soc., 85, 381-394.

Santanello, J. A., M. A. Friedl, and M. B. Ek, 2007: Convective planetary boundary layer interactions with the land surface at diurnal time scales: Diagnostics and feedbacks. J. Hydrometeor., 8, 1082-1097.

—, C. D. Peters-Lidard, S. V. Kumar, C. Alonge, and W.-K. Tao, 2009: A modeling and observational framework for diagnosing local land-atmosphere coupling on diurnal time scales. J. Hydrometeor., 10, 577-599.

Schlosser, C. A., and P. R. Houser, 2007: Assessing a satellite-era perspective of the global water cycle. J. Climate, 20, 1316-1338.

— , and Coauthors, 2000: Simulations of a boreal grassland hydrology at Valdai, Russia: PILPS phase 2(d). Mon. Wea. Rev., 128, 301-321.

Sellers, P. J., Y. Mintz, Y. C. Sud, and A. Dalcher, 1986: A Simple Biosphere Model (SIB) for use within general circulation models. J. Atmos. Sci., 43, 505-531.

Seneviratne, S. I., D. Luthi, M. Litschi, and C. Schar, 2006: Land-atmosphere coupling and climate change in Europe. Nature, 443, 205-209. 
_, T. Corti, E. L. Davin, M. Hirschi, E. B. Jaeger, I. Lehner, B. Orlowsky, and A. J. Teuling, 2010: Investigating soil moisture-climate interactions in a changing climate: A review. Earth-Sci. Rev., 99, 125-161.

Slater, A. G., and Coauthors, 2001: The representation of snow in land-surface schemes: Results from PILPS 2(d). J. Hydrometeor., 2, 7-25.

Van den Hurk, B. J. J. M., and P. Viterbo, 2003: The Torne-Kalix PILPS2e experiment as testbed for modifications to the ECMWF land surface scheme. Global Planet. Change, 38, 165-173.

—, and E. M. Blyth, 2008: WATCH/LoCo workshop report. GEWEX Newsletter, No. 18, International GEWEX Project Office, Silver Spring, MD, 12-14.

Wood, E. F., and Coauthors, 1998: The Project for Intercomparison of Land-Surface Parameterization Schemes (PILPS) phase 2(c) Red-Arkansas River basin experiment: 1. Experiment description and summary intercomparisons. Global Planet. Change, 19, 115-135. 